\title{
Reflexiones sobre la maternidad durante la adolescencia
}

\author{
Reflections on motherhood during adolescence
}

Yamila Mahumud $^{*}$, Vilda Discacciati ${ }^{\ddagger}$ y Sergio Terrasa ${ }^{5}$

Mahumud Y*, Discacciati V ${ }^{\ddagger}$, Terrasa S. ${ }^{\S}$ Reflexiones sobre la maternidad durante la adolescencia. Evid Act Pract Ambul. 2014 17(4). Oct-Dic 118-119.

La adolescencia es un tiempo de crecimiento y desarrollo, con adquisición de nuevas funciones, desde el aspecto biológico, psicológico y social ${ }^{\dagger+1}$. Es un periodo crucial del ciclo vital en el que los individuos toman una nueva dirección en su desarrollo, alcanzan la madurez sexual, y se apoyan en los recursos psicosociales desarrollados previamente en el seno de su grupo de origen, intentando elaborar su propia identidad y formar un proyecto de vida propio.

Cuando nos referimos a que la adolescencia es el periodo en el cual los individuos intentan desarrollar su identidad, sabemos que la sociedad dificulta este proceso, sobre todo para las mujeres adolescentes. Esto estaría vinculado con que históricamente, la sociedad tiende a determinarles lugares subordinados en lo que respecta a la división sexual de funciones y roles, privilegiando los relacionados con la maternidad ${ }^{2 \ddagger \ddagger}, y$ salvo excepciones, asociados con una baja participación social e inserción laboral.

En relación a la sexualidad, la posición de la sociedad es ambigua, ya que por un lado los adolescentes reciben estímulos sexuales desde los medios de comunicación masivos, se les reconoce el derecho a mantener relaciones sexuales y a acceder a los medios anticonceptivos, y por otro, reciben la mirada condenatoria de sus mayores cuando sufren las consecuencias de una sexualidad no responsable, como el embarazo no deseado o las enfermedades de transmisión sexual.

Desde el ideario social de quienes pertenecemos a clases sociales medias o altas, cuando hablamos de "proyecto de vida", solemos asociarlo con estudiar o trabajar para poder mejorar las condiciones de vida en el futuro, en los que la paternidad, la maternidad y la formación de la familia, si bien pueden ocupar un espacio importante, no son considerados eventos imprescindibles y además, están pensados para una etapa bastante posterior a la adolescencia, fundamentalmente por las aspiraciones de movilidad social ascendente. En cualquiera de estos casos y especialmente si se trata de adolescentes provenientes de hogares pobres y/o con pocos recursos familiares $y / 0$ sociales, se sabe que un hijo puede alterar ese proyecto, ya sea postergándolo o directamente representando la imposibilidad de llevarlo a cabo ${ }^{35}$, con la consiguiente pérdida de oportunidades para estos jóvenes, que se convierte prematuramente en padres y para su hijo, que se verá arrojado a este mundo en unos brazos que quizás todavía no estén preparados para criarlo.

El embarazo en la adolescencia es un fenómeno biopsicosocial que tiene consecuencias muy importantes, tanto para los y las adolescentes como para sus hijos ${ }^{\star \star *}$. Es conocido que tanto la maternidad como la paternidad son consideradas funciones propias de la edad adulta, independientemente de las características propias de la adolescencia en cada cultura y del significado que el embarazo pueda tener en cada una de ellas ${ }^{\dagger t}$. Además, existe una tendencia cultural en este momento histórico, a considerar que el embarazo y la maternidad/paternidad durante la adolescencia no son adecuados, independientemente de que se produzcan o no efectos adversos en la salud, y más allá de la edad o del contexto de cada adolescente embarazada y de si dicho embarazo fue resultado de un abuso o buscado y/o deseado.

La sexualidad humana es variable y se encuentra muy influenciada por factores sociales y culturales que repercuten en la probabilidad de que una adolescente se embarace y en el significado que este puede llegar a tener para ella. Cometeríamos un error si diéramos por sentado que la totalidad de los embarazos que ocurren en la adolescencia son "accidentes" o no intencionales, ya que parte de la fecundidad que ocurre durante la adolescencia sucede en el contexto de parejas estables que realmente estaban buscando ese embarazo.

Para muchas jóvenes la maternidad sería un medio para reafirmar su deseo de salir adelante y/o la posibilidad de tener un hijo como "algo propio". También puede interpretarse como la expresión de conflictos no resueltos (fallecimiento o pérdida de seres queridos) o del deseo de dejar de estudiar, o bien como la transición hacia otro estatus social. En contextos marcados por desigualdades de género y clase, la maternidad se presenta no sólo como destino sino más bien como una fuente de reconocimiento social, sobre todo para las adolescentes desprovistas de proyectos educativos y profesionales ${ }^{4}$.

Así, como vemos que el embarazo en esta etapa de la vida puede ser aceptado de diversas maneras y según el contexto en el que suceda, la crianza del hijo también varía en cada circunstancia. No es lo mismo criar un hijo que "es natural que llegue", que a otro que representa "la posibilidad de salir de un medio familiar violento", o a aquel que venga a "avergonzar a la familia", o al que "condicione a la madre a postergar sus estudios o proyectos personales".

La tasa de fecundidad adolescente promedio en el mundo se estima en 49,7 por mil para el período 2000-05 pero está lejos de reflejar la diversidad existente en diferentes regiones $\$ \neq+$. Lo mismo ocurre en Argentina, donde el $10,7 \%$ de las adolescentes censadas en 2001 eran madres, con importantes diferencias entre las jurisdicciones $\$ \S$

Parte de las investigaciones desarrolladas en América Latina ${ }^{6}$ revelan que una proporción considerable de los jóvenes tiene

\footnotetext{
* Servicio de Medicina Familiar y Comunitaria del Hospital Italiano de Buenos Aires.
}

₹Servicio de Medicina Familiar y Comunitaria del Hospital Italiano de Buenos Aires y Departamento de Salud Pública del Instituto Universitario Hospital Italiano de Buenos Aires. § Servicio de Medicina Familiar y Comunitaria y Departamento de Investigación del Hospital Italiano de Buenos Aires, y Departamento de Salud Pública del Instituto Universitario Hospital Italiano de Buenos Aires.

"t Según la Organización Mundial de la Salud (OMS), la adolescencia corresponde a la etapa de transición entre la infancia y la edad adulta, comprendida entre los 10 y los 19 años de edad.

\# Como adolescentes, la sociedad las ubica en un grupo etario caracterizado por ser transicional entre la niñez y la adultez.

$\$ \$$ Por ejemplo, un estudio descriptivo realizado durante 2007 en Perú a través de la entrevista de 120 embarazadas adolescentes con el objetivo de determinar las condiciones personales y las expectativas futuras de desarrollo personal que se asocian a dicha condición mostró que las entrevistadas iniciaron precozmente las relaciones sexuales, que en el $81 \%$ el embarazo no había sido deseado, que el $84 \%$ tenía información acerca de métodos anticonceptivos y que el $38 \%$ los había utilizado. Vale destacar que el $72 \%$ se encontraba estudiando previamente y que sólo $24 \%$ continuó haciéndolo.

*** La expresión "embarazo adolescente" denota inmediatamente una valoración negativa y se entiende como un problema público sobre el cual la opinión de los adultos -padres, maestros, médicos, comunicadores, y políticos- tiene tanto o mayor peso que la de los propios jóvenes involucrados.

†t† Una adolescente no madura a la adultez por el simple hecho de estar embarazada. Seguirá siendo una adolescente que tendrá que hacerse cargo de su hijo y le será mucho más difícil lograr las metas que alguna vez se haya planteado -como por ejemplo estudiar una carrera o trabajar en lo que desee, o quizás casarse-, ya que deberá dedicarse a la crianza de su hijo. La maternidad a edades precoces también puede verse agravada por el impacto socio-familiar que el embarazo genera, en ocasiones reacciones de rechazo por parte del grupo familiar de la adolescente embarazada o por parte de su pareja. 
escasos conocimientos sobre sexualidad y reproducción, carece de información suficiente sobre anticoncepción, tropieza con obstáculos cuando intenta acceder a métodos de planificación familiar y tiene grandes dificultades para adoptar medidas de protección en sus prácticas sexuales, quedando expuesta al riesgo de contraer enfermedades de transmisión sexual (ETS) o a embarazarse sin quererlo ${ }^{7}$.

Más allá de que numerosísimas investigaciones ${ }^{8,9}$, establecieron que los factores asociados al embarazo adolescente se pueden clasificar en individuales, familiares y sociales ${ }^{\star \star \star *}$; consideramos pertinente explayarnos en los resultados de un estudio basado en grupos focales con adolescentes de diferentes regiones de Argentina ${ }^{10}$. Fue realizado con el objetivo de conocer las actitudes y conductas de los adolescentes en relación al embarazo en la adolescencia y su impacto sobre ellos y su entorno. Los investigadores concluyeron que el embarazo que sucede en esta etapa de la vida generalmente es no buscado y consecuencia de la no utilización de métodos anticonceptivos o de su uso incorrecto o irregular. Interpretaron estos comportamientos asociados a la sensación de "inmunidad" que experimentan los adolescentes, con la consiguiente exposición a conductas de riesgo (aun sabiendo las consecuencias), o con una postura pasiva frente a la posibilidad de un embarazo, en la que se lo entiendo como algo que "simplemente sucede" o "pasa sin querer".

La concepción del embarazo en la adolescencia como "problema" de salud pública ${ }^{11}$, merecedor de atención en términos de investigación y de intervención por parte del Estado ${ }^{t+t+}$, surge en la década del sesenta del siglo XX, acompañando los cambios sociodemográficos, económicos, culturales y políticos que tuvieron lugar tras la Segunda Guerra Mundial ${ }^{12}$.

Sin embargo, consideramos que caracterizar el embarazo adolescente como "problema" sin cuestionar las estructuras de desigualdad y los procesos de vulnerabilización que vienen afectando a los adolescentes y los jóvenes en general, es por lo menos simplista. Algunos estudios que han puesto el foco de atención en el impacto a largo plazo de la maternidad adolescente ${ }^{13,14}$, sugieren que centrar el debate exclusivamente en las estrategias basadas en la postergación de la paternidad, sin ofrecer modificaciones sustanciales en las oportunidades de educación y en las perspectivas que como sociedad se les ofrece a los jóvenes (independientemente del ámbito social al que pertenezcan) no será capaz de modificar la problemática de la perpetuación de la pobreza en general y del embarazo adolescente en particular. Se podría presuponer que en las clases sociales de menores recursos, el problema estaría en la falta de información acerca de métodos anticonceptivos o en el difícil acceso a estos, lo que se relacionaría con la baja escolaridad que habitualmente se ve en estos sectores. O también podemos pensar que para estas adolescentes, tener un hijo y formar una familia puede ser el único proyecto de vida al que pueden aspirar. Si esto fuera así, el objetivo fundamental de las políticas públicas -y parte de nuestro desafío como profesionales de la salud y como ciudadanos-, debería centrarse en la ejecución de estrategias de prevención destinadas a las adolescentes que no son madres, a las que están embarazadas y a las madres adolescentes y sus hijos, con el objetivo de favorecer la generación y el mantenimiento del vínculo entre ellos, de ayudarlas para que puedan ejercer una planificación familiar con autonomía, y de fomentar y promover la reinserción educativa y laboral de la madre. Consideramos que es el momento de poner el foco de atención en promover el desarrollo personal y profesional de los adolescentes, alentándolos y brindándoles un contexto favorable para que continúen estudiando, y de esta manera, puedan lograr llevar a cabo el proyecto que cada uno en particular se esté proponiendo.

Recibido en 02/05/14 y aceptado el 03/01/15.

\section{Referencias}

1. Peláez J. Embarazo en la adolescencia. Ginecología Infanto Juvenil. La Habana. 1999: 190-202.

2. Climent G. La maternidad adolescente: una expresión de la cuestión social, el interjuego entre la exclusión social, construcción de la subjetividad y políticas públicas. Revista Argentina de Sociología №1, 2003, p 77-93.

3. Langer A El embarazo no deseado: impacto sobre salud y sociedad en América Latina y Caribe. Revista Panamericana de Salud Pública 11 (3) 2002.

4. Binstock $G$ y col. La fecundidad adolescente hoy: diagnostico socio-demografico. 2006.

5. Climent $\mathrm{G}$ y col. Maternidad adolescente hoy: un camino hacia la marginación. Cuadernos Médicos Sociales 77: 81-101.

6. Cogna M. Reproductive Health Matters 2008; 16 (31):192-201.

7. Szames $\mathrm{C}$ y col. Conocimiento, utilización y obtención de la anticoncepción hormonal de emergencia por adolescentes en Rauch, Provincia de Buenos Aires.Rev Argent Salud Pública, Vol. 3 - No 11, Junio 2012.

8. Langer L y col. Decision- making orientation and AIDS-related knowledge, attitudes, and white adolescents. Health Psychology, 3:227-234

9. Moore $\mathrm{K}$ y col. Begining too soon: adolescent sexual behavior, pregnancy and parenthood. A review of research and interventions. Publicaciones electronicas de PAHO, 32:12-15.

10. Zamberlin N. Percepciones y conductas de las/los adolescentes frente al embarazo y la maternidad/paternidad. Centro de Estudios de Estado y Sociedad (CEDES), 2000. 11. Allca Moreno G. Adolescente embarazada: condiciones personales y expectativas futuras de desarrollo personal. Hospital María Auxiliadora 2007. Revista Peruana de Obstetricia 5 (2) 2009

12. Coll A. Embarazo en la adolescencia: ¿Cuál es el problema? Adolescencia y Juventud en América Latina, Libro Universitario Regional, Cartago 2001.

13. Flórez C. Factores socioeconómicos y contextuales que determinan la actividad reproductiva de las adolescentes en Colombia. Revista Panamericana de Salud Publica.2005; 18(6): 388-402.

14. Alonso Uría R y col. Embarazo en la adolescencia: algunos factores biopsicosociales. Revista Cubana de Medicina General Integral Vol. 21, № 5-6, Septiembre-Diciembre 2005.

\# Los países con niveles mayores a 200 por mil (Liberia, Níger, Sierra Leone, Somalia y Uganda) se ubican en África donde la tasa de fecundidad adolescente promedio supera los 100 por mil. En Asia ese promedio se estima en 35 por mil, un valor mucho menor que el de África, semejante al de Oceanía (32,3 por mil) y bastante superior al de Europa (20,3 por mil). El promedio asiático esconde la existencia de países con tasas superiores a 100 por mil (Afganistán, Bangladesh, Nepal, Yemen) y países con los niveles más bajos de fecundidad adolescente del mundo (Japón, China, las dos Coreas, Singapur), similares a los de algunos países europeos como Holanda, Suiza, Italia, España y Suecia, entre otros, todos con tasas entre el 3 y el 6 por mil. Con un nivel muy similar al promedio mundial se ubica Estados Unidos (53,2 por mil) y por arriba de dicho promedio se encuentra el de América Latina y el Caribe (72,4 por mil), región en la cual conviven países con tasas superiores a 100 (Guatemala, Honduras y Nicaragua) con otros con fecundidad adolescente relativamente baja (Guadalupe, Martinica) y varios en un rango intermedio. La Argentina con un nivel estimado de fecundidad adolescente de 60,6 por mil está por debajo de sus vecinos Bolivia, Brasil, Paraguay y Uruguay, y por encima de Chile.

${ }^{\$ \varsigma}{ }^{\mathrm{L}} \mathrm{La}$ Ciudad de Buenos Aires es la jurisdicción que muestra niveles más bajos de madres adolescentes (4\%), seguida por Mendoza, con más del doble (8,5\%). En contraste, Chaco es la provincia con índices más altos (17\%), seguida por Santiago del Estero, Misiones y Catamarca, con un promedio de $15 \%$ de madres adolescentes.

**** Respecto a lo individual, los factores asociados son: inicio cada vez más precoz de la actividad sexual, menarca a edades menores, sensación de invulnerabilidad, baja autoestima, impulsividad, mala relación con los padres y carencia de proyectos educacionales. En el plano familiar, se asocia a baja escolaridad de los padres, mala relación afectiva, padre o madre ausente, violencia en la resolución de conflictos familiares, padres permisivos o poco accesibles y a que la madre se haya embarazado en la adolescencia. Y finalmente, en cuanto al ámbito social, se pudieron identificar diversas variables asociadas a la actividad sexual precoz, con el consiguiente aumento del embarazo adolescente: erotización de los medios masivos de comunicación, estrés, hacinamiento, residencia en áreas rurales, y los todavía aun presentes "mitos y tabúes" acerca de la sexualidad, lo que contribuye a la escasa información acerca de la reproducción humana, acerca de los diferentes métodos anticonceptivos y sobre los factores emocionales relacionados con la sexualidad humana.

$+1+t$ La Organización Mundial de la Salud reconoce al embarazo adolescente como un embarazo de riesgo en el que se afecta la salud tanto de la madre como del embrión en desarrollo, ya que se sabe está asociado a un mayor índice de complicaciones durante el embarazo y el parto (anemia, desgarros del canal de parto, endometritis, infecciones del sitio quirúrgico, mastitis, etc.) y que generalmente se acompaña de condiciones adversas. 